\title{
Human miRNome Profiling in Colon Adenoma-carcinoma Sequence Reveal Early Deregulation of miR-1246, Which Exhibits Oncogenic Properties in Vitro by Targeting AXIN2 and CFTR
}

\section{Simonas Juzenas}

Lithuanian University of Health Sciences

\section{Rokas Lukosevicius}

Lithuanian University of Health Sciences

Violeta Salteniene

Lithuanian University of Health Sciences

Ugne Kulokiene

Lithuanian University of Health Sciences

Georg Hemmrich-Stanisak

Christian-Albrechts-University of Kiel

\section{Andre Franke}

Christian-Albrechts-University of Kiel

Alexander Link

Otto-von-Guericke University

\section{Paulius Ruzgys}

Vytautas Magnus University

\section{Saulius Satkauskas}

Vytautas Magnus University

Henrikas Pauzas

Lithuanian University of Health Sciences

Tadas Latkauskas

Lithuanian University of Health Sciences

\section{Gediminas Kiudelis}

Lithuanian University of Health Sciences

\section{Francesc Balaguer}

Hospital Clínic Barcelona, University of Barcelona

\section{Juozas Kupcinskas}

Lithuanian University of Health Sciences

Jurgita Skieceviciene ( $\sim$ jurgita.skieceviciene@lsmuni.lt )

Lithuanian University of Health Sciences

\section{Research Article}

Keywords: Colorectal cancer, Adenomatous polyps, MicroRNA, IsomiR, Small RNA-seq

Posted Date: June 2nd, 2021

DOI: https://doi.org/10.21203/rs.3.rs-558860/v1 
License: (c) (i) This work is licensed under a Creative Commons Attribution 4.0 International License. Read Full License 


\section{Abstract}

Background: Regulatory changes occurring early in colorectal cancer development remain poorly investigated. Since the majority of cases develop from polyps in the adenoma-carcinoma pathway, a search of early molecular features, such as aberrations in miRNA expression occurring prior to cancer development, would enable identification of potentially causal, rather than consequential, candidates in the progression of polyp to cancer. This study aim was to discover early miRNA expression changes in adenoma-carcinoma sequence and to investigate the role of deregulated miRNAs in CRC development.

Methods: Small RNA-seq data analysis was performed to identify deregulated miRNAs and their isoforms among HC, AP and CRC tissue samples. Deregulated miRNAs were validated using RT-qPCR in the independent patient group. MTT, colony formation and wound healing assays were performed in order to evaluate hsa-miR-1246 function in CRC cells. Luciferase and Western blot assays were used to test hsa-miR-1246 gene targets.

Results: In the current study by employing small RNA-seq profiling of colon biopsy samples, we describe differentially expressed miRNAs and their isoforms in the adenoma-carcinoma pathway. Analysis of healthy-adenoma-carcinoma sequence in an independent validation group enabled to identify early deregulated miRNAs including hsa-miR-1246 and hsa-miR-215-5p and up-regulation of hsa-miR-1246 in plasma samples of patients with CRC. Loss-of-function experiments revealed that inhibition of hsa-miR-1246 leads to reduced cell viability, colony formation and migration rate, thereby indicating an oncogenic effect of this miRNA in vitro. Subsequent western blot and luciferase reporter assay provided evidence of hsa-miR-1246 being involved in the regulation of target $A X I N 2$ and $C F T R$ genes' expression.

Conclusions: The present study revealed possible involvement of hsa-miR-1246 in early colorectal cancer development and regulation of tumor suppressors AXIN2 and CFTR.

\section{Background}

Colorectal cancer (CRC) is currently one of the most prevalent cancer types worldwide and is the second leading cause of cancer-related mortality [1]. Generally, from 70 to $90 \%$ of CRC cases arise from the traditional adenoma-carcinoma pathway, where conventional adenomas (polyps) progress by the sequential accumulation of genetic mutations and chromosomal instability causing microsatellite stable adenocarcinomas [2]. In the adenoma-carcinoma pathway, the above-mentioned alterations inactivate tumor-suppressor genes and activate oncogenes leading to cancer stem cell formation, which is essential for CRC initiation, growth and maintenance [2, 3]. Multiple genetic, transcriptional and functional studies have identified numerous coding genes that affect viability, growth and migration of CRC cells [4]. These findings, besides providing important aspects of CRC pathogenesis also contributed to the development of novel therapeutic targets (e.g., anti-VEGF and anti-EGFR monoclonal antibodies [5]) and patient stratification strategies [6]. However, the effectiveness of these approaches has been proven only in the subsets of patients, thus disease management success remains poor and requires new defined molecular features. Therefore, investigating the regulatory mechanisms that occur prior to cancer stem cell development, at a polyp stage, is a promising field of CRC research for possible therapeutic agents, preventive treatment and diagnostic biomarkers.

In addition to the genetic variability and differential expression of coding genes among tumor cells, noncoding RNAs and specific epigenetic marks further contribute to functional tumor heterogeneity [3]. For example, microRNAs (miRNAs) are known to regulate different aspects of tumorigenesis, from tumor initiation to the maintenance of established tumors [7]. These small RNAs mediate translational repression of messenger RNAs (mRNAs) through 3'UTR-specific antisense interactions [8] and, based on their function in cancerogenesis, can act either as oncogenes or tumor suppressors [9]. Although miRNAs are annotated and cataloged as single defined sequences, alternative processing choices (incl. alternative Drosha and/or Dicer mediated cleavage, non-templated nucleotide addition and RNA editing) along the miRNA biogenesis pathway can generate multiple miRNA isoforms (isomiRs) which can expand the regulatory repertoire of miRNA genes and either enhance or reduce their cancerogenicity $[8,10]$. It has been shown that miRNAs (incl. their isoforms) are deregulated in nearly all cancer types, including CRC [11-16] and might be used as biomarkers [17] as well as therapeutic targets [18] in various diseases. Therefore, molecular profiling and functional examination of aberrantly expressed miRNAs along the adenoma-carcinoma pathway is 
needed to further understand the pathogenesis of CRC and to identify putative targets for treatment or even diagnostics of the disease.

In this study, using small RNA-seq profiling of colon biopsy samples from healthy individuals and patients with CRC or colon adenomas, we describe differentially expressed miRNAs and their isoforms in the adenoma-carcinoma pathway. In addition to this, by utilizing RT-qPCR validation in the independent group of patients we report a robust, early and gradual deregulation of hsa-miR-1246 and hsa-miR-215-5p in the adenoma-carcinoma pathway and up-regulation of hsa-miR-1246 in plasma samples of patients with CRC. Finally, by using functional assays, we show that hsa-miR-1246 exhibits an oncogenic effect in vitro and provide evidence of its involvement in the direct regulation of AXIN2 and CFTR gene expression.

\section{Methods}

\section{Study population}

Tissue and plasma samples from patients with CRC (colorectal cancer) or AP (adenomatous polyps) and HC (healthy control) subjects were prospectively collected at the Department of Gastroenterology and Department of Surgery, Lithuanian University of Health Sciences (Kaunas, Lithuania) during 2011-2014 years. The specimens of all investigated groups were biopsies, taken from affected or healthy colorectal tissues during routine colonoscopy or surgical tumor removal. Biopsies of the CRC group were histologically verified as being colorectal adenocarcinomas. The preneoplastic lesion group was comprised of advanced adenoma patients (conventional adenomas with high-grade dysplasia, adenomas of $>1 \mathrm{~cm}$ diameter or villous histology). The HC group consisted of healthy subjects, who underwent colonoscopy due to positive fecal occult blood test (FOBT), but had no history of previous malignancy and were otherwise healthy. All patients included in the study were of European descent. Clinical and phenotypic characteristics of subjects investigated in profiling and validation cohorts are presented in Table 1.

\section{RNA isolation}

Total RNA (including small RNA fraction) was extracted from snap-frozen tissue and blood plasma samples using miRNeasy Mini Kit (Qiagen, MD, USA) and miRNeasy Serum and Plasma Kit (Qiagen, USA), respectively, according to the recommendations of the manufacturer. The quality and quantity of the isolated RNA were assessed by Nanodrop 2000 spectrophotometer (Thermo Fisher Scientific, MA, USA) or by Agilent 2100 Bioanalyzer (Agilent Technologies, CA, USA).

\section{Small RNA library preparation and NGS}

Small RNA libraries were generated with $1 \mu \mathrm{g}$ RNA input per sample applying a standard TruSeq Small RNA Sample Preparation Kit (Illumina, CA, USA) protocol. Agilent 2100 Bioanalyzer (Agilent Technologies, USA) was used for quality and yield assessment of sequencing libraries. Small RNA libraries were randomly selected, pooled of approximately 24 samples/lane and sequenced $(1 \times 50$ bp single-end reads) on HiSeq2500 (Illumina, USA) NGS platform.

\section{Small RNA-Seq data analysis}

The generated raw sequencing reads (fastq) were processed by cutadapt v1.9 [19] which was used to trim adapter sequences and low-quality bases (< Q20) as well as discard sequences shorter than $18 \mathrm{nt}$ in length. The processed small RNA-seq reads were assigned to miRBase v21, using quantifier.pl [20] and miraligner.jar [21] for reference miRNAs and isomiRs, respectively. In order to reduce false-positives in isomiR sequence substitutions, a mismatch was considered as a real substitution when the minimum fraction rate of reads having that substitution was equal to 0.25 . Additionally, only the isomiRs with uniquely mapped substitutions were kept for downstream analyses. Generated miRNA/isomiR counts were normalized using size factor normalization and variance stabilizing transformation (VST) employing the DESeq2 package [22]. The abundance differences of isomiR variations among the $\mathrm{CRC}, \mathrm{AP}$, and $\mathrm{HC}$ groups were examined by the Kruskal-Wallis test. Differential expression analyses of miRNAs were performed by employing negative binomial generalized linear models and Wald statistics implemented in the DESeq2 R package. The models were fitted using technical batch and age (centered) as covariates, since the average age was significantly different $(P<0.05)$ between $C R C$ and $\mathrm{HC}$ groups. Benjamini and Hochberg correction of false discovery rate (FDR) was used for P-value adjustment. The miRNAs with $\mathrm{P}_{\mathrm{FDR}}<0.01$ and absolute value of log2 fold change 
$(\log 2 \mathrm{FC})>0.5$ were considered as significantly differentially expressed. Such log2FC and $\mathrm{P}_{\mathrm{FDR}}$ value threshold was chosen to detect mild changes in expression $(0.5<\log 2 \mathrm{FC}<1)$, that are considerably consistent $\left(\mathrm{P}_{\mathrm{FDR}}<0.01\right)$ among patients within group. Results of three-way differential expression analysis were visualized using the volcano3D R package [23]. Spearman's rank correlation coefficient was used for correlation analysis. The raw sequencing data, as well as miRNA counts, have been deposited at the Gene Expression Omnibus (GEO) under the accession number of GSE160432 (link: www.ncbi.nlm.nih.gov/geo/query/acc.cgi?acc=GSE160432; reviewer token: upcdwguslrglfwx).

\section{RT-qPCR validation analysis}

Quantification of hsa-miR-1246 and hsa-miR-215-5p expression in tissue samples of CRC, AP and healthy individuals were analyzed using TaqMan ${ }^{\circledR}$ MicroRNA Assays (Applied Biosystems, CA, USA) according to the producer's recommendations in technical duplicates. MiRNA expression in plasma samples was quantified in two batches - hsa-miR-1246 and hsa-miR-215-5p expression in CRC and healthy controls plasma samples was quantified by using TaqMan® MicroRNA Assays (Applied Biosystems, USA), while expression in AP and the same healthy controls plasma samples was analyzed by using TaqMan ${ }^{\circledR}$ Advanced MicroRNA Assays (Applied Biosystems, USA). Accordingly, the results of miRNA expression in plasma of CRC and AP groups were analyzed separately. Endogenous hsa-miR-16-5p (tissue and plasma) and hsa-miR-191-5p (plasma) were used as internal normalizers in both tissue and plasma samples $[24,25]$. The expression levels of hsa-miR-1246 and hsa-miR-215-5p were normalized using $\Delta C T$ method [26]. The mean value of duplicate $\Delta C T$ values of each sample was submitted to the nonparametric Mann-Whitney $U$ test. The miRNAs with a false discovery rate (FDR) corrected $\mathrm{P}<0.05$ and an absolute value of log2 fold change $>1$ were considered as significantly differentially expressed.

\section{Target gene set enrichment analysis}

Target gene set enrichment analysis for hsa-miR-1246 and hsa-miR-215 was accomplished using miTALOS v2 [27] tool. The tool was used to perform overrepresentation analysis of biological pathways with default settings using TargetScan miRNAtarget collection and employing Fisher's exact test as well as Benjamini-Hochberg multiple test correction procedure. Pathways with $\mathrm{P}_{\mathrm{FDR}}<0.05$ and enrichment score $(\mathrm{E})>1$ were considered as significantly overrepresented among hsa-miR-1246 and/or hsa-miR-215 target genes.

\section{Cell culture}

Human colorectal adenocarcinoma SW620 (CCL-227 ${ }^{\mathrm{TM}}$, ATCC® ${ }^{\text {, VA, USA), Caco-2 (HTB-37 }}{ }^{\mathrm{TM}}$, ATCC $\circledast$, USA), HCT116 (CCL-247 ${ }^{\mathrm{TM}}$, ATCC $\AA$, USA), and gastric adenocarcinoma AGS (CRL-1739 ${ }^{\mathrm{Tm}}$, ATCC $\AA$, USA) cell lines were cultured in Ham's F-12K (Kaighn's) Medium (Gibco, MA, USA) supplemented with 10\% fetal bovine serum (Gibco, USA) and 1\% penicillin/streptomycin (100 U/mL penicillin and $100 \mathrm{mg} / \mathrm{mL}$ streptomycin, Corning, NY, USA), in a humidified incubator at $37^{\circ} \mathrm{C}$ with $5 \% \mathrm{CO}_{2}$.

\section{Cell transfection}

For colony formation, MTT, wound healing, luciferase and protein expression assays, reverse transfection with hsa-miR-1246 inhibitor (Assay ID: MH13182, mirVana ${ }^{T M}$, CA, USA) or mimic (Assay ID: MC13182, mirVana ${ }^{T M}$, USA) and miR-Control (mirVana ${ }^{\text {TM }}$, USA) (final concentration $100 \mathrm{pmol} / \mathrm{ml}$ ) was performed using lipofectamine 3000 reagent (Invitrogen, CA, USA).

\section{Wound healing assay}

Reverse transfected HCT116 and Caco-2 cells were seeded into a 2-well silicone insert with a defined cell-free gap (Ibidi, Gräfelfing, Germany) for the wound healing assay $\left(4 \times 10^{4}\right.$ cells per well). Before the insert removal, the cells were cultured until reaching $90 \%$ confluency. The formed gap was captured at four different time points every $24 \mathrm{~h}$ with an inverted light microscope (Olympus IX71, Tokyo, Japan). The ratio between the remaining and the initial size of the wound area was evaluated using Image-J software (MD, USA). At least three independent experiments were performed.

\section{MTT assay}

MTT assay was used for the assessment of the metabolic activity of the cells, which reflects the quantity of viable cells. Reverse transfected SW620 and Caco-2 cells were seeded into a 96-well plate (5000 cells per well). 3-(4,5-dimethylthiazol-2- 
yl)-2,5-diphenyltetrazolium bromide (MTT; Sigma-Aldrich Co., MO, USA) (final concentration $0.5 \mathrm{mg} / \mathrm{ml}$ ) was added into each well $72 \mathrm{~h}$ after the seeding. Cells were incubated with MTT reagent for another 2 hours and after that, the medium with reagent was removed. Formed formazan crystals were dissolved using dimethyl sulfoxide (DMSO; Carl Roth GmbH + Co., Karlsruhe, Germany) (200 UI). Light absorbance was detected with Sunrise plate reader (Tecan, Männedorf, Switzerland) at $570 \mathrm{~nm}$ wavelength and a reference wavelength at $620 \mathrm{~nm}$. At least three independent experiments were performed.

\section{Colony formation assay}

Both reverse transfected Caco-2 and SW620 cell lines were seeded into a 6-well plate (250 cells per well). After 2 weeks of incubation with complete medium, the cells were washed with PBS, fixed with 10\% formaldehyde (Sigma Aldrich, Germany) for 20 min and stained with 1\% crystal violet (Alfa Aesar by Thermo Fisher Scientific, Kandel, Germany) for 15 min at room temperature. The number of colony-forming units was calculated using ImageJ software (USA) in three independent experiments.

\section{Target prediction}

Putative target genes, having 8-mer binding sites for hsa-miR-1246 were retrieved from the TargetScan v7.2 [28] database. Since the hsa-miR-1246 showed increased expression levels (oncogenic) in CRC, the targets were selected based on their function in carcinogenesis, i.e., targets having tumor-suppressive function were chosen for further investigation.

\section{Protein extraction and Western Blot}

Reverse transfected SW620 and Caco-2 cells were seeded in 6-well plates $\left(1 \times 10^{6}\right.$ cells per well) for protein expression experiments. $72 \mathrm{~h}$ after transfection, cells were lysed using $1 \times$ radioimmunoprecipitation assay (RIPA) buffer (Abcam, Cambridge, UK) containing phosphatase and protease inhibitor cocktail (Sigma Aldrich, USA). Total protein concentration was evaluated using Pierce BCA Protein Assay Kit (Thermo Fisher Scientific, USA). The extracted protein was fractionated by SDSPAGE using 4-12\% Bis-Tris Plus Mini Gels and transferred to $0.45 \mu \mathrm{m}$ PVDF membrane. PVDF membranes were blocked using WesternBreeze ${ }^{\mathrm{TM}}$ Blocker/Diluent (Part A and Part B) (Thermo Fisher Scientific, USA) at room temperature for $1 \mathrm{~h}$. Antibodies directed against AXIN2 (1:1000 dilution; Cat. No. ab109307; Abcam, UK), CFTR (1:500 dilution; Cat. No. ab2784, Abcam, UK) and GAPDH $(0.4 \mu \mathrm{g} / \mathrm{ml}$ concentration; Cat. No. AM4300; Ambion by Thermo Fisher Scientific, CA, USA) were used. Protein signals were detected and visualized using ChemiDocTM XRS + System (Bio-Rad, CA, USA) and ImageLab Software v5.2.1 (Bio-Rad, USA). GAPDH protein was used as an endogenous control. At least three independent experiments were performed.

\section{Dual light luciferase assay}

Wild-type (WT) and mutant (MT) seed region sites of hsa-miR-1246 containing 3'UTR sequence of target genes were constructed and cloned into pMIR-REPORT-Luciferase vector (Invitrogen, USA) (Fig. 3B). Constructed insert sequences were verified by Sanger sequencing using BigDye Terminator v3.1 kit (Applied Biosystems, USA) and 3500 Series Genetic Analyzer (Applied Biosystems, USA). Previously well-established workflow for dual luciferase assay using AGS cells was applied for this experiment $[29,30]$. Cells were co-transfected with hsa-miR-1246 mimic and miR-Control, pMIR-REPORT-Luciferase-WT vector, PMIR-REPORT-Luciferase-MT vector, and PMIR-REPORT-ß-galactosidase control vector (Invitrogen, USA). Luciferase activity was detected after $48 \mathrm{~h}$ of incubation by the Dual-Light ${ }^{\text {TM }}$ Luciferase \& $\beta$-Galactosidase Reporter Gene Assay System (Invitrogen, USA) and normalized with ß-galactosidase activity using Tecan Genios Pro (Tecan, Switzerland). At least three independent experiments were performed.

\section{Statistical analysis}

All data from the functional experiments in CRC cell lines are given as means \pm standard deviation (SD) of at least three independent experiments. Data between groups were compared using a two-tailed Student's t-test for the normally distributed data or the Mann-Whitney U test for the not normally (shown by Shapiro-Wilk test) distributed data. All statistical calculations were performed with R Studio software version v3.6.0 (MA, USA). False discovery rate (FDR) corrected $\mathrm{P}<0.05$ was considered statistically significant.

\section{Results}




\section{Small RNA-Seq defines altered expression of miRNAs and isomiRs along adenoma-carcinoma sequence}

To evaluate whether the changes in miRNA expression occur in the transition from normal colon tissue to adenoma and from adenoma to adenocarcinoma, small RNA-Seq was performed on tissue biopsies of CRC, AP, and HC individuals. The global similarity of miRNA transcriptomes was estimated using multidimensional scaling analysis (MDS) on VST normalized miRNA count data (see Methods). The analysis showed three clearly resolved clusters concordant with CRC, AP, and HC groups. The AP cluster was an intermediate between $\mathrm{CRC}$ and HC groups. To be precise, the AP cluster showed high overlap with CRC on the first MDS (MDS1) dimension and high overlap with HC on the second MDS (MDS2) dimension (Fig. 1A), which suggests transitional changes in global miRNA expression levels from normal mucosa to adenocarcinoma.

To explore whether the variability within miRNA sequences is also reflected in the adenoma-carcinoma pathway, the analysis of isomiRs (variations at the 5' and 3' ends, nucleotide (nt) substitutions and non-templated $3^{\prime}$ additions) was performed. The overall abundances of isomiR modification types were unevenly distributed $\left(P_{F D R}<0.05\right)$ across the groups, except for $3^{\prime}$ addition (Supplementary Fig. 1 A). Interestingly, the abundances of 3 ' addition and seed shifting (5'-trimming) modifications were significantly gradually decreasing in polyp to cancer sequence (from HC to CRC) at positions 1 and - 1 (with respect to reference miRNA), respectively (Supplementary Fig. 1B and 1C). These results show that some types of miRNA modifications are distributed in a transitional fashion, where AP tissue samples have intermediate values between CRC and HC groups.

To further define differences in miRNA and their isoform expression among CRC, AP, and HC tissues, differential expression analysis was performed. The analysis identified 208 differentially expressed miRNAs, 157 of which were up-regulated and 51 were down-regulated in CRC when compared to HC, whereas 146 miRNAs were found to be deregulated in AP compared to HC. The majority of those were up-regulated $(n=89)$ in AP and only a number of miRNAs $(n=57)$ were down-regulated. Differential expression analysis between CRC and AP identified 191 altered miRNAs, 122 of which were up-regulated and 69 were downregulated (Supplementary Table 1). The results of deregulated isomiRs in the analogous comparisons are provided in Supplementary Table 2. The three-way differential expression analysis of both colon conditions (CRC and AP) against HC revealed that the expression levels of 60 miRNAs (such as hsa-miR-1246, hsa-miR-31-5p and hsa-miR-135b-5p) were commonly elevated in the AP and CRC tissue samples when compared to HC. In CRC, 67 miRNAs were uniquely up-regulated, whereas in the AP alone, the expression of 37 distinct miRNAs (including hsa-miR-509-3p and hsa-miR-423-5p) were increased. Interestingly, $\mathrm{HC}$ and $\mathrm{CRC}$ had more commonly up-regulated miRNAs ( $\mathrm{n}=49$; including hsa-miR-7-5p and hsa-miR-9-5p) compared to commonly upregulated miRNAs ( $n=27$; including hsa-miR-215-5p and hsa-miR-375) in HC and AP groups (Fig. 1B; Supplementary Table 3).

Taken together, the results of small RNA-seq show a high overlap between CRC and AP miRNA transcriptomes and reflect AP being the intermediate condition between CRC and HC.

\section{Expression levels of hsa-miR-1246 and hsa-miR-215-5p are gradually and robustly changing along the adenoma-carcinoma pathway}

To identify miRNAs that correlate with adenoma to carcinoma pathway, Spearman's rank correlation analysis was performed by treating transitional disease stages as ordinal values. Two miRNAs (hsa-miR-1246 and hsa-miR-215-5p) highly correlated (| $r$ Spearman's $1>0.7$ ) with the adenoma-carcinoma sequence. Gradual up-regulation of hsa-miR-1246 was determined in colorectal polyps and cancer when compared to healthy mucosa, whereas hsa-miR-215-5p was gradually down-regulated in the same sequence (Fig. 1C; Supplementary Table 4). The analysis also identified 28 moderately correlating $\left(0.5<\left|r_{\text {Spearman's }}\right|<0.7\right)$ miRNAs with adenoma-carcinoma sequence, 21 of which were gradually up-regulated and 7 were gradually down-regulated (Supplementary Fig. 2; Supplementary Table 4).

To further evaluate whether the highly correlating hsa-miR-1246 and hsa-miR-215-5p are robustly differentially expressed along adenoma-carcinoma sequence, the RT-qPCR validation was performed in colon tissues of an independent cohort comprised of 
120 individuals $(C R C=40 ; A P=40 ; H C=40)$ (Table 1). Differential expression analyses showed that both of the miRNAs were deregulated in CRC and AP tissue when compared to HC in the same direction as in the NGS data (Fig. 1D).

To get more insights and to test whether the expression changes of these two miRNAs can be detected in the body fluids of the patients, the second validation step was performed in plasma samples of the same cohort $(n=120)$ using RT-qPCR method. The analysis revealed that the normalized expression levels of hsa-miR-1246 were significantly $\left(P_{F D R}<0.05\right)$ up-regulated in CRC when compared to $\mathrm{HC}$, while the expression levels of hsa-miR-215-5p were not deregulated in CRC and AP plasma samples (Fig. 1D). To further determine the possible biological impact of hsa-miR-215-5p and hsa-miR-1246 in CRC pathogenesis, target gene set enrichment analysis (GSEA) was performed. In total, 5 Reactome pathways were significantly enriched $\left(P_{F D R}<0.05\right.$ and $E>1$ ) in hsa-miR-215-5p and/or hsa-miR-1246 target genes, 3 out of which were responsible for "Signaling by WNT in cancer" (both miRNAs: $P_{F D R}=2.12 \times 10^{-3}$; hsa-miR-215-5p: $P_{F D R}=0.015$ ), "Degradation of beta-catenin by the destruction complex" (both miRNAs: $P_{F D R}=2.72 \times 10^{-5}$ ), and "Binding of TCF/LEF:CTNNB1 to target gene promoters" (hsa-miR-1246: $P_{F D R}$ $=0.024)$. Other two significantly enriched pathways for hsa-miR-1246 targets were "CAMP signaling pathway" $\left(P_{F D R}=0.03\right.$; KEGG) and "BDNF signaling pathway" ( $P_{\text {FDR }}=0.04$; WikiPathways) (Supplementary Fig. 3). 
Table 1

Clinical and phenotypic information of profiling and validation cohorts.

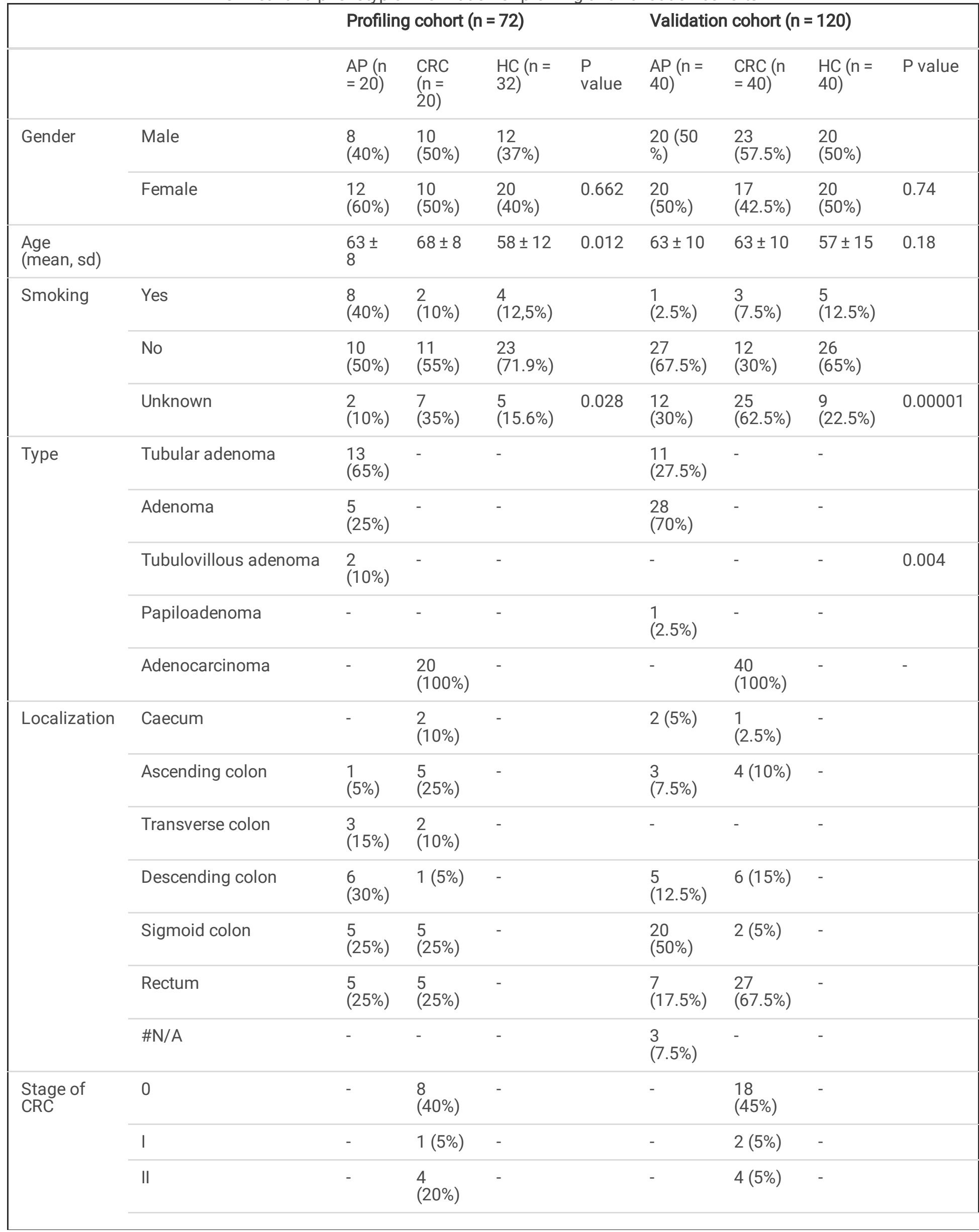

Page 9/19 


\begin{tabular}{|c|c|c|c|c|c|c|c|}
\hline \multirow[b]{2}{*}{ III } & \multicolumn{3}{|c|}{ Profiling cohort $(n=72)$} & \multicolumn{4}{|c|}{ Validation cohort $(n=120)$} \\
\hline & - & $\begin{array}{l}4 \\
(20 \%)\end{array}$ & - & - & $\begin{array}{l}14 \\
(35 \%)\end{array}$ & - & \\
\hline IV & - & $\begin{array}{l}3 \\
(15 \%)\end{array}$ & - & - & $2(5 \%)$ & - & 0.451 \\
\hline
\end{tabular}

Categorical variables are summarized as absolute numbers and percentages (in parentheses), while age (quantitative variable) is summarized as mean and standard deviation. To test for differences among groups, Chi squared test was used for categorical variables, while Kruskal-Wallis test was used for quantitative variables. AP: Adenomatous polyp patients; CRC: Colorectal cancer patients; HC: Healthy controls.

\section{Inhibition of hsa-miR-1246 reduced cell viability, colony formation and migration rate in CRC cells}

Since hsa-miR-1246 showed gradual up-regulation in AP and CRC tissues and up-regulation in CRC plasma, suggesting a possible oncogenic effect, it was selected for further functional characterization. To test this hypothesis, MTT and colony formation assays were performed to evaluate the effect of hsa-miR-1246 on cell viability and proliferation. The MTT assay revealed that inhibition of hsa-miR-1246 reduced the viability of Caco-2 $(26.3 \%, P=0.02)$ and SW620 $(26.67 \%, P=0.002)$ cells $72 \mathrm{~h}$ after transfection (Fig. 2A). In addition, the inhibition of hsa-miR-1246 reduced colony number formed by Caco-2 cells (43.3\%, $P=0.03$ ) while no differences were found in SW620 cells $(P>0.05)$ (Fig. 2B).

The effect of hsa-miR-1246 on CRC cell migration was examined using a wound-healing assay. Complete coverage (100\%) of the gap area was reached $72 \mathrm{~h}$ after cell transfection with both miR-Control and hsa-miR-1246 inhibitor in Caco-2 cell line and $96 \mathrm{~h}$ after transfection with miR-Control and hsa-miR-1246 inhibitor in HCT116 cell line. Inhibition of hsa-miR-1246 significantly slowed gap covering in Caco-2 and HCT116 cells $24 \mathrm{~h}$ (Caco-2: 9.79\%, $\mathrm{P}=0.018$; HCT116: 6.55\%, $\mathrm{P}=0.01)$ and $48 \mathrm{~h}$ (Caco-2: $8.24 \%, \mathrm{P}=0.04$; HCT116: 8.82\%, $\mathrm{P}=0.007$ ) after transfection when compared to miR-Control (Fig. $2 \mathrm{C}$ ).

Collectively, the results of the functional tests in CRC-originated cells support the potential oncogenic function of hsa-miR-1246 in CRC.

\section{hsa-miR-1246 suppresses the expression of CFTR and AXIN2 by directly targeting their 3'UTR}

Considering that cell migration and proliferation rates were reduced by the inhibition of hsa-miR-1246, the predicted target genes were also selected based on their function and possible involvement in the WNT signaling pathway, which regulates cell fate determination, cell migration and cell polarity [31]. More precisely, using in silico predictions, targets of hsa-miR-1246 containing 8-mer binding sites were retrieved from TargetScan database and literature searched for known function in oncogenesis [27, 32]. Predicted target genes for hsa-miR-1246, namely AXIN2 and CFTR have met the selection criteria and were chosen for the downstream analysis of protein expression. Inhibition of hsa-miR-1246 increased levels of AXIN2 and CFTR proteins in Caco-2 and SW620 cells $72 \mathrm{~h}$ after transfection with anti-miR-1246. Level of CFTR protein was higher in both Caco-2 (53\%, P = 0.02) and SW620 (32\%, P = 0.04) cells, while AXIN2 was significantly higher only in SW620 (68\%, P = 0.02) cells, compared to the negative control (miR-Control) (Fig. 3A).

To test whether the observed upregulation of AXIN2 and CFTR proteins is due to the direct interaction between hsa-miR-1246 and target (3'UTR) sequences, their 3'UTR fragments corresponding to the putative miRNA target sites (wild-type or mutated) were cloned into a luciferase vector (Fig. 3B). The constructed vectors together with hsa-miR-1246 mimics or negative controls were transfected into cells. The transfection of hsa-miR-1246 mimics significantly reduced wt-CFTR and wt-AXIN2 luciferase reporter activity compared to negative miRNA control (miR-Control) (Fig. 3C). The reporter activity did not change in plasmids containing mt-CFTR and mt-AXIN2 constructs after hsa-miR-1246 mimic and miR-Control transfection. These findings demonstrate that hsa-miR-1246 specifically targets CFTR (position 1537-1544 of 3' UTR) and AXIN2 (position 313-320 of 3' UTR) transcripts by binding to their target sites. These findings together with the results of the functional tests in CRC cells 
support the hypothesis that increased levels of hsa-miR-1246 might enhance translational repression of AXIN2 and CFTR genes, both of which are members of WNT signaling pathway.

\section{Discussion}

Majority of CRC pathologies derive from a polyp in a so-called traditional adenoma-carcinoma pathway, where an aberrant colon crypt evolves into an adenomatous polyp and eventually progresses to CRC [2]. This process not only involves sequential acquisition of genetic alterations, but also epigenetic aberrations such as uncontrolled expression of regulatory miRNAs [33]. For this reason, a search of early regulatory changes occurring at a polyp stage provides a possibility to identify putative candidates that are potentially causal, rather than consequential in the progression of adenoma to cancer.

By following this logic, small RNA-seq profiling was performed on colon tissue samples from healthy individuals and patients with colon adenomas or with CRC. Subsequent exploratory analysis on similarity structure of obtained miRNomes revealed colonic adenomas as being an intermediate condition between healthy colon mucosa and adenocarcinoma, which reflected the sequential transition of polyp to cancer in the adenoma-carcinoma pathway and confirmed miRNA impairment at early stages of the disease that have already been shown in previous studies [34]. Based on the statistical estimates of differential expression analysis, numerous miRNAs were found to be deregulated along the adenoma-carcinoma sequence. Most of them, including hsa-miR-1246, hsa-miR-215-5p, hsa-miR-31, hsa-miR-135b-5p and hsa-miR-375 have been previously shown to be deregulated in CRC [16, 35-38], underlining their possible involvement in cancerogenesis. Moreover, in our dataset hsa-miR1246 and hsa-miR-215-5p showed the highest correlation with the adenoma-carcinoma pathway - the former displayed a gradual increase, while the latter showed a gradual decrease in expression levels along the heathy-adenoma-carcinoma sequence, which is partly in line with a previous study by Nagy et al [34]. Nagy et al. study used microarray (discovery) and RTPCR (validation) approaches to identify deregulated miRNAs in adenoma to cancer sequence. The major limitation of this study is that they used the same patient group in the discovery and validation steps and hsa-miR-215-5p failed to be replicated due to technical issues, whereas hsa-miR-1246 showed significant upregulation only through the normal to adenoma. In our study, these findings were validated in the independent group of patients, proving robust and early deregulation of hsa-miR-1246 and hsa-miR-215-5p, thereby suggesting their possible involvement in the early stages of carcinogenesis.

It has already been shown that hsa-miR-215-5p is frequently downregulated in CRC tissues [36-38] and exhibits tumor suppressor activity through the regulation of EGFR or WNT signaling pathways [37, 39]. In the case of hsa-miR-1246, a persistent increase of its expression levels was previously reported in both tissue and plasma samples of CRC [40,41], which agrees with our findings as well. Although the origin of hsa-miR-1246 remains controversial due to sequence overlap with the central region of the RNU2-1 transcript [42], this molecule has been repeatedly reported to function as an oncogene and to promote tumor angiogenesis, growth, migration, invasion, metastasis and stemness in various cancer types, including CRC [40, 42-45]. Our results are in compliance with the aforementioned reports and showed that inhibition of hsa-miR-1246 reduced viability and migration rates of CRC cells, confirming the oncogenic function of hsa-miR-1246.

Selection of in silico predicted hsa-miR-1246 target genes was based on the facts acquired from transcriptional and functional parts of this study and involved tumor suppressors participating in a signaling pathway that regulates cell fate determination or cell migration, such as WNT signaling pathway [31]. The genes AXIN2 and CFTR have met these criteria and were selected for further investigation. $A X I N 2$ gene is known to play an important role in WNT/ $\beta$-catenin signaling pathway through phosphorylation and degradation of $\beta$-catenin [46]. CFTR gene encodes a chloride channel that controls ion and water secretion as well as absorption in epithelial tissues. Although the exact mechanism is unclear, intestinal tumor tissue analysis of CFTR knockout mice showed increased $\beta$-catenin levels and revealed functional implication of CFTR in WNT pathway regulation [27]. It was already known that CRC tumors frequently contain mutations in WNT pathway components such as APC, CTNNB1, and/or AXIN2 genes, which cause aberrant WNT activation [47]. Recent studies have shown the linkage between the hsa-miR1246 and WNT signaling pathway and its function in lung and oral carcinogenesis $[48,49]$. Our data revealed that inhibition of hsa-miR-1246 enhanced levels of AXIN2 and CFTR tumor suppressor proteins in CRC cells. The luciferase assay verified that this effect is caused by direct 3'UTR-specific interaction between their mRNAs and the seed site of hsa-miR-1246, confirming that both AXIN2 and CFTR genes are targets of hsa-miR-1246. This is partly in line with the results by Chai et al., who reported 
AXIN2 as a direct target of hsa-miR-1246 in liver cancer cells [50]. The regulation of these WNT-associated targets by hsa-miR1246 could also explain the different effect on cell proliferation rate (number colonies formed) in Caco-2 and SW620 cells. This may occur due to uneven deregulation of pathways responsible for cell line proliferation such as WNT signaling pathway [31] since it has been previously reported that SW620 cell line shows stronger WNT signaling than Caco-2 cells [51]. Our results are also supported by the previous observations that AXIN2 and CFTR are frequently downregulated in CRC and are both linked with poor prognosis of the patients [27, 52-54]. Moreover, it has been shown that inactivating mutations in CFTR gene increased the early incidence and progression of colon adenomas [55]. While we do not claim to fully understand the function of hsa-miR1246, altogether, our data, as well as other studies, provide sufficient evidence of hsa-miR-1246 involvement in early CRC cancerogenesis, possibly, via AXIN2 and CFTR gene expression regulation. The identification of early regulatory changes of hsamiR-1246 expression has the potential to be further investigated in bigger cohorts for disease monitoring and prognosis application of adenoma progression to carcinoma. Moreover, the impact of hsa-miR-1246 inhibition on cell viability, growth and migration should be pursued in vivo in future studies to assess its possible therapeutic modality, at least in CRC.

This study has certain limitations that need to be acknowledged. The enrolled CRC patients have not been tested for KRAS and $B R A F$ mutations and other types of molecular phenotyping have not been performed. Furthermore, due to the sample size, we were unable to evaluate the association of miRNAs with survival and treatment response; however, we did not have an aim to address these issues in our study design and rather focused on general molecular aspects of the adenoma-carcinoma pathway. Nevertheless, we believe that our present study has significance in adding additional knowledge on the role of miRNAs in the adenoma-carcinoma pathway.

\section{Conclusion}

In conclusion, we showed gradual deregulation of hsa-miR-1246 and hsa-miR-215-5p in the colon adenoma-carcinoma pathway. We also found that hsa-miR-1246 exhibits an oncogenic effect in vitro with an effect on cell viability, colony formation and migration in CRC cell lines. Finally, we provide evidence of hsa-miR-1246 being involved in AXIN2 and CFTR gene expression regulation which might affect the WNT signaling pathway and would be the scope of our future explorations.

\section{Abbreviations}

miRNA (miR): microRNA; RNA-seq: RNA sequencing; AXIN2: gene coding Axin-like protein; AXIN2: Axin-like protein; CFTR: cystic fibrosis transmembrane conductance regulator protein; CFTR: gene coding Cystic fibrosis transmembrane conductance regulator; CRC: colorectal cancer; Anti-VEGF: vascular endothelial growth factor protein antibody; Anti-EGFR: epidermal growth factor receptor protein antibody; 3'UTR: 3' untranslated region; RT-qPCR: Reverse Transcription quantitative polymerase chain reaction; AP: adenomatous polyp; FOBT: fecal occult blood test; NGS: next generation sequencing; isomiR: microRNA isoform;

HC: healthy control; GEO: gene expression omnibus; PBS: phosphate-buffered saline; RIPA: radioimmunoprecipitation assay; PVDF: polyvinylidene difluoride membrane; GAPDH: Glyceraldehyde 3-phosphate dehydrogenase protein; WT: wild-type; MT: mutant-type; MDS: multidimensional scaling analysis; VST: variance stabilizing transformation; EGFR: epidermal growth factor receptor protein; RNU2-1:, RNA U2 small nuclear 1; APC., adenomatous polyposis coli gene; CTNNB1:, gene Catenin Beta 1 protein; KRAS:, gene coding Kirsten rat sarcoma viral oncogene homolog; $B R A F \mathrm{~B}: B R A F$, gene coding B-Raf protein.

\section{Declarations}

\section{Ethics approval and consent to participate:}

The use of biological material in the present study was approved by the Kaunas Regional Biomedical Research Ethics Committee (protocol no. BE-2-10). Each study subject has signed written informed consent and all procedures were carried out in accordance with the guidelines of Declaration of Helsinki.

\section{Consent for publication:}

Not applicable. 
Availability of data and materials:

The raw sequencing data, as well as miRNA counts, that support the findings of this study, are available in the Gene Expression Omnibus (GEO), reference number GSE160432 (Link: www.ncbi.nlm.nih.gov/geo/query/acc.cgi?acc=GSE160432; reviewer token: upcdwguslrglfwx). Other data that supports the findings of this study are available in the supplementary material of this article or are available from the corresponding author upon reasonable request.

\section{Competing interests:}

The authors declare no competing interests.

\section{Funding:}

This study was supported by the Research Council of Lithuania under the initiative of Researcher Group Projects, grant no. SMIP-17-22.

\section{Authors' contributions:}

Juzenas S, Kupcinskas J, Skieceviciene J designed and coordinated the study; Juzenas S, Lukosevicius R, Salteniene V accomplished experiments; Juzenas S, Lukosevicius R performed data analysis and interpretation and statistical analysis; Juzenas S, Lukosevicius R, Salteniene V, Kulokiene U wrote original draft; Skieceviciene J, Kupcinskas J, Balaguer F, Link A reviewed and edited the manuscript; Juzenas S, Lukosevicius R made visualizations; resources: Kiudelis G, Pauzas H, Latkauskas T, Hemmrich-Stanisak G, Franke A, Satkauskas S, Ruzgys P - resources; Skieceviciene J, Kupcinskas J supervision. All authors approved the final version of the article.

\section{Acknowledgements:}

Not applicable.

\section{References}

1. Bray F, Ferlay J, Soerjomataram I, Siegel RL, Torre LA, Jemal A. Global cancer statistics 2018: GLOBOCAN estimates of incidence and mortality worldwide for 36 cancers in 185 countries. CA Cancer J Clin. 2018;68:394-424.

2. Dekker E, Tanis PJ, Vleugels JLA, Kasi PM, Wallace MB. Colorectal cancer. Lancet (London, England). 2019;394:1467-80. doi:10.1016/S0140-6736(19)32319-0.

3. Nassar D, Blanpain C. Cancer Stem Cells: Basic Concepts and Therapeutic Implications. Annu Rev Pathol. 2016;11:47-76. doi:10.1146/annurev-pathol-012615-044438.

4. Kuipers EJ, Grady WM, Lieberman D, Seufferlein T, Sung JJ, Boelens PG, et al. Colorectal cancer. Nat Rev Dis Prim. 2015;1:15065. doi:doi: 10.1038/nrdp.2015.65.

5. Venook AP, Niedzwiecki D, Lenz H-J, Innocenti F, Fruth B, Meyerhardt JA, et al. Effect of First-Line Chemotherapy Combined With Cetuximab or Bevacizumab on Overall Survival in Patients With KRAS Wild-Type Advanced or Metastatic Colorectal Cancer: A Randomized Clinical Trial. JAMA. 2017;317:2392-401. doi:10.1001/jama.2017.7105.

6. Muzny DM, Bainbridge MN, Chang K, Dinh HH, Drummond JA, Fowler G, et al. Comprehensive molecular characterization of human colon and rectal cancer. Nature. 2012;487:330-7. doi:10.1038/nature11252.

7. Lin S, Gregory RI. MicroRNA biogenesis pathways in cancer. Nat Rev Cancer. 2015;15:321-33. doi:10.1038/nrc3932.

8. Bartel DP. Metazoan MicroRNAs. Cell. 2018;173:20-51. doi:10.1016/j.cell.2018.03.006.

9. Esquela-Kerscher A, Slack FJ. Oncomirs - microRNAs with a role in cancer. Nat Rev Cancer. 2006;6:259-69. doi:10.1038/nrc1840.

10. Dhanoa JK, Verma R, Sethi RS, Arora JS, Mukhopadhyay CS. Biogenesis and biological implications of isomiRs in mammals- a review. ExRNA. 2019;1:3. doi:10.1186/s41544-018-0003-8. 
11. Hofsli E, Sjursen W, Prestvik WS, Johansen J, Rye M, Tranø G, et al. Identification of serum microRNA profiles in colon cancer. Br J Cancer. 2013;108:1712-9. doi:10.1038/bjc.2013.121.

12. Matsumura $T$, Sugimachi $K$, linuma $H$, Takahashi $Y$, Kurashige J, Sawada G, et al. Exosomal microRNA in serum is a novel biomarker of recurrence in human colorectal cancer. Br J Cancer. 2015;113:275-81. doi:10.1038/bjc.2015.201.

13. Du L, Zhang X, Li J, Wang C, Yang X, Zheng G, et al. Serum microRNA panel as biomarkers for early diagnosis of colorectal adenocarcinoma. Br J Cancer. 2014;111:1985-92.

14. Kanth P, Bronner MP, Boucher KM, Burt RW, Neklason DW, Hagedorn CH, et al. Gene signature in sessile serrated polyps identifies colon cancer subtype. Cancer Prev Res. 2016;9:456-65.

15. Telonis AG, Magee R, Loher P, Chervoneva I, Londin E, Rigoutsos I. Knowledge about the presence or absence of miRNA isoforms (isomiRs) can successfully discriminate amongst 32 TCGA cancer types. Nucleic Acids Res. 2017;45:2973-85. doi:10.1093/nar/gkx082.

16. Wu CW, Evans JM, Huang S, Mahoney DW, Dukek BA, Taylor WR, et al. A Comprehensive Approach to Sequence-oriented IsomiR annotation (CASMIR): demonstration with IsomiR profiling in colorectal neoplasia. BMC Genomics. 2018;19:401. doi:10.1186/s12864-018-4794-7.

17. Carter J V, Galbraith NJ, Yang D, Burton JF, Walker SP, Galandiuk S. Blood-based microRNAs as biomarkers for the diagnosis of colorectal cancer: a systematic review and meta-analysis. Br J Cancer. 2017;116:762-74.

doi:10.1038/bjc.2017.12.

18. Forterre A, Komuro H, Aminova S, Harada M. A Comprehensive Review of Cancer MicroRNA Therapeutic Delivery Strategies. Cancers (Basel). 2020;12. doi:10.3390/cancers12071852.

19. Martin M. Cutadapt removes adapter sequences from high-throughput sequencing reads. EMBnet.journal. 2011;17:10-2.

20. Friedländer MR, Mackowiak SD, Li N, Chen W, Rajewsky N. miRDeep2 accurately identifies known and hundreds of novel microRNA genes in seven animal clades. Nucleic Acids Res. 2012;40:37-52. doi:10.1093/nar/gkr688.

21. Pantano L, Estivill X, Marti E. SeqBuster, a bioinformatic tool for the processing and analysis of small RNAs datasets, reveals ubiquitous miRNA modifications in human embryonic cells. Nucleic Acids Res. 2010;38:e34-e34. doi:10.1093/nar/gkp1127.

22. Love MI, Huber W, Anders S. Moderated estimation of fold change and dispersion for RNA-seq data with DESeq2. Genome Biol. 2014;15:550. doi:10.1186/s13059-014-0550-8.

23. Lewis MJ, Barnes MR, Blighe K, Goldmann K, Rana S, Hackney JA, et al. Molecular Portraits of Early Rheumatoid Arthritis Identify Clinical and Treatment Response Phenotypes. Cell Rep. 2019;28:2455-2470.e5. doi:10.1016/j.celrep.2019.07.091.

24. Zheng $G$, Wang $H$, Zhang $X$, Yang $Y$, Wang L, Du L, et al. Identification and validation of reference genes for qPCR detection of serum microRNAs in colorectal adenocarcinoma patients. PLoS One. 2013;8:e83025. doi:10.1371/journal.pone.0083025.

25. Wang L, Liu Y, Du L, Li J, Jiang X, Zheng G, et al. Identification and validation of reference genes for the detection of serum microRNAs by reverse transcription-quantitative polymerase chain reaction in patients with bladder cancer. Mol Med Rep. 2015;12:615-22. doi:10.3892/mmr.2015.3428.

26. Livak KJ, Schmittgen TD. Analysis of relative gene expression data using real-time quantitative PCR and the 2(-Delta Delta C(T)) Method. Methods. 2001;25:402-8. doi:10.1006/meth.2001.1262.

27. Than BLN, Linnekamp JF, Starr TK, Largaespada DA, Rod A, Zhang Y, et al. CFTR is a tumor suppressor gene in murine and human intestinal cancer. Oncogene. 2016;35:4191-9. doi:10.1038/onc.2015.483.

28. Agarwal V, Bell GW, Nam J-W, Bartel DP. Predicting effective microRNA target sites in mammalian mRNAs. Elife. 2015;4:e05005. doi:10.7554/eLife.05005.

29. Gyvyte U, Lukosevicius R, Inciuraite R, Streleckiene G, Gudoityte G, Bekampyte J, et al. The role of MiR-375-3p and MiR200b-3p in gastrointestinal stromal tumors. Int J Mol Sci. 2020;21:1-12. doi:10.3390/ijms21145151.

30. Streleckiene G, Inciuraite R, Juzenas S, Salteniene V, Steponaitiene R, Gyvyte U, et al. Mir-20b and mir-451a are involved in gastric carcinogenesis through the PI3K/AKT/mTOR signaling pathway: Data from gastric cancer patients, cell lines and 
ins-gas mouse model. Int J Mol Sci. 2020;21. doi:10.3390/ijms21030877.

31. Komiya Y, Habas R. Wnt signal transduction pathways. Organogenesis. 2008;4:68-75. doi:10.4161/org.4.2.5851.

32. Kandimalla R, Linnekamp JF, van Hooff S, Castells A, Llor X, Andreu M, et al. Methylation of WNT target genes AXIN2 and DKK1 as robust biomarkers for recurrence prediction in stage II colon cancer. Oncogenesis. 2017;6:e308-e308. doi:10.1038/oncsis.2017.9.

33. Schetter AJ, Okayama H, Harris CC. The role of microRNAs in colorectal cancer. Cancer J. 2012;18:244-52. doi:10.1097/PPO.0b013e318258b78f.

34. Nagy ZB, Wichmann B, Kalmár A, Galamb O, Barták BK, Spisák S, et al. Colorectal adenoma and carcinoma specific miRNA profiles in biopsy and their expression in plasma specimens. Clin Epigenetics. 2017;9:22. doi:10.1186/s13148-016-0305-3.

35. Fromm B, Høye E, Böttger PHM, Domanska D, Kristensen AT, Lund-Andersen C, et al. Towards a consensus microRNA signature of primary and metastatic colorectal cancer. bioRxiv. 2020;:2020.06.01.127647. doi:10.1101/2020.06.01.127647.

36. Karaayvaz M, Pal T, Song B, Zhang C, Georgakopoulos P, Mehmood S, et al. Prognostic Significance of miR-215 in Colon Cancer. Clin Colorectal Cancer. 2011;10:340-7. doi:10.1016/j.clcc.2011.06.002.

37. Jones MF, Hara T, Francis P, Li XL, Bilke S, Zhu Y, et al. The CDX1-microRNA-215 axis regulates colorectal cancer stem cell differentiation. Proc Natl Acad Sci U S A. 2015;112:E1550-8. doi:10.1073/pnas.1503370112.

38. Zhang J-X, Song W, Chen Z-H, Wei J-H, Liao Y-J, Lei J, et al. Prognostic and predictive value of a microRNA signature in stage II colon cancer: a microRNA expression analysis. Lancet Oncol. 2013;14:1295-306. doi:10.1016/S14702045(13)70491-1.

39. Vychytilova-Faltejskova P, Merhautova J, Machackova T, Gutierrez-Garcia I, Garcia-Solano J, Radova L, et al. MiR-215-5p is a tumor suppressor in colorectal cancer targeting EGFR ligand epiregulin and its transcriptional inducer HOXB9. Oncogenesis. 2017;6:399. doi:10.1038/s41389-017-0006-6.

40. Wang S, Zeng Y, Zhou JM, Nie SL, Peng Q, Gong J, et al. MicroRNA-1246 promotes growth and metastasis of colorectal cancer cells involving CCNG2 reduction. Mol Med Rep. 2016;13:273-80.

41. Ogata-Kawata H, Izumiya M, Kurioka D, Honma Y, Yamada Y, Furuta K, et al. Circulating Exosomal microRNAs as Biomarkers of Colon Cancer. PLoS One. 2014;9:e92921. doi:10.1371/journal.pone.0092921.

42. Xu Y-F, Hannafon BN, Khatri U, Gin A, Ding W-Q. The origin of exosomal miR-1246 in human cancer cells. RNA Biol. 2019;16:770-84. doi:10.1080/15476286.2019.1585738.

43. Chai S, Ng KY, Tong M, Lau EY, Lee TK, Chan KW, et al. Octamer 4/microRNA-1246 signaling axis drives Wnt/ $\beta$-catenin activation in liver cancer stem cells. Hepatology. 2016;64:2062-76.

44. Yamada N, Tsujimura N, Kumazaki M, Shinohara H, Taniguchi K, Nakagawa Y, et al. Colorectal cancer cell-derived microvesicles containing microRNA-1246 promote angiogenesis by activating Smad 1/5/8 signaling elicited by PML downregulation in endothelial cells. Biochim Biophys Acta. 2014;1839:1256-72. doi:10.1016/j.bbagrm.2014.09.002.

45. Sun Z, Meng C, Wang S, Zhou N, Guan M, Bai C, et al. MicroRNA-1246 enhances migration and invasion through CADM1 in hepatocellular carcinoma. BMC Cancer. 2014;14:616. doi:10.1186/1471-2407-14-616.

46. Jho E, Zhang T, Domon C, Joo C-K, Freund J-N, Costantini F. Wnt/\$ $\beta$ \$-Catenin/Tcf Signaling Induces the Transcription of Axin2, a Negative Regulator of the Signaling Pathway. Mol Cell Biol. 2002;22:1172-83. doi:10.1128/mcb.22.4.11721183.2002.

47. Novellasdemunt L, Antas P, Li VSW. Targeting Wnt signaling in colorectal cancer. A review in the theme: Cell signaling: Proteins, pathways and mechanisms. Am J Physiol - Cell Physiol. 2015;309:C511-21. doi:10.1152/ajpcell.00117.2015.

48. Yang F, Xiong H, Duan L, Li Q, Li X, Zhou Y. MiR-1246 Promotes Metastasis and Invasion of A549 cells by Targeting GSK3 $\beta$-Mediated Wnt/ $\beta$-Catenin Pathway. Cancer Res Treat. 2019;51:1420-9. doi:10.4143/crt.2018.638.

49. Lin SS, Peng CY, Liao YW, Chou MY, Hsieh PL, Yu CC. miR-1246 targets CCNG2 to enhance cancer stemness and chemoresistance in oral carcinomas. Cancers (Basel). 2018;10. doi:10.3390/cancers10080272.

50. Chai S, Ng KY, Tong M, Lau EY, Lee TK, Chan KW, et al. Octamer 4/microRNA-1246 signaling axis drives Wnt/ $\beta$-catenin activation in liver cancer stem cells. Hepatology. 2016;64:2062-76. doi:10.1002/hep.28821. 
51. Christensen J, El-Gebali S, Natoli M, Sengstag T, Delorenzi M, Bentz S, et al. Defining new criteria for selection of cell-based intestinal models using publicly available databases. BMC Genomics. 2012;13. doi:10.1186/1471-2164-13-274.

52. Picco G, Petti C, Centonze A, Torchiaro E, Crisafulli G, Novara L, et al. Loss of AXIN1 drives acquired resistance to WNT pathway blockade in colorectal cancer cells carrying RSPO3 fusions. EMBO Mol Med. 2017;9:293-303.

doi:10.15252/emmm.201606773.

53. Chen HY, Lang YD, Lin HN, Liu YR, Liao CC, Nana AW, et al. miR-103/107 prolong Wnt/\$\$\$-catenin signaling and colorectal cancer stemness by targeting Axin2. Sci Rep. 2019;9. doi:10.1038/s41598-019-41053-z.

54. Tan GC, Chan E, Molnar A, Sarkar R, Alexieva D, Isa IM, et al. 5 ' isomiR variation is of functional and evolutionary importance. Nucleic Acids Res. 2014;42:9424-35. doi:10.1093/nar/gku656.

55. Niccum DE, Billings JL, Dunitz JM, Khoruts A. Colonoscopic screening shows increased early incidence and progression of adenomas in cystic fibrosis. J Cyst Fibros. 2016;15:548-53. doi:10.1016/j.jcf.2016.01.002.

\section{Figures}

A

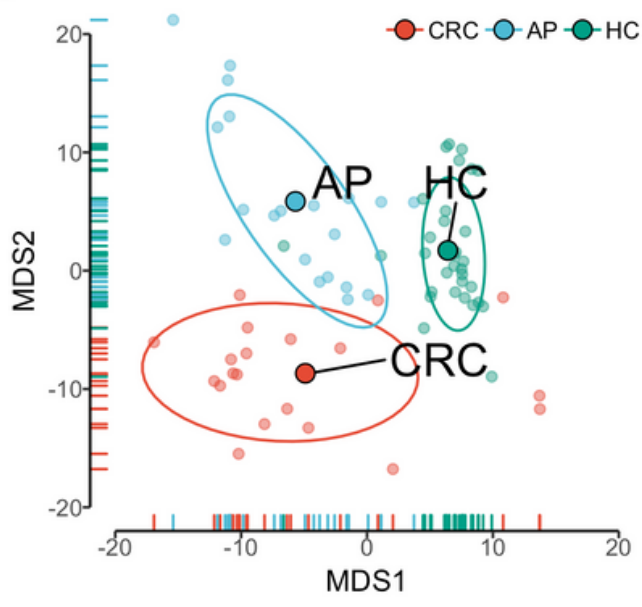

B

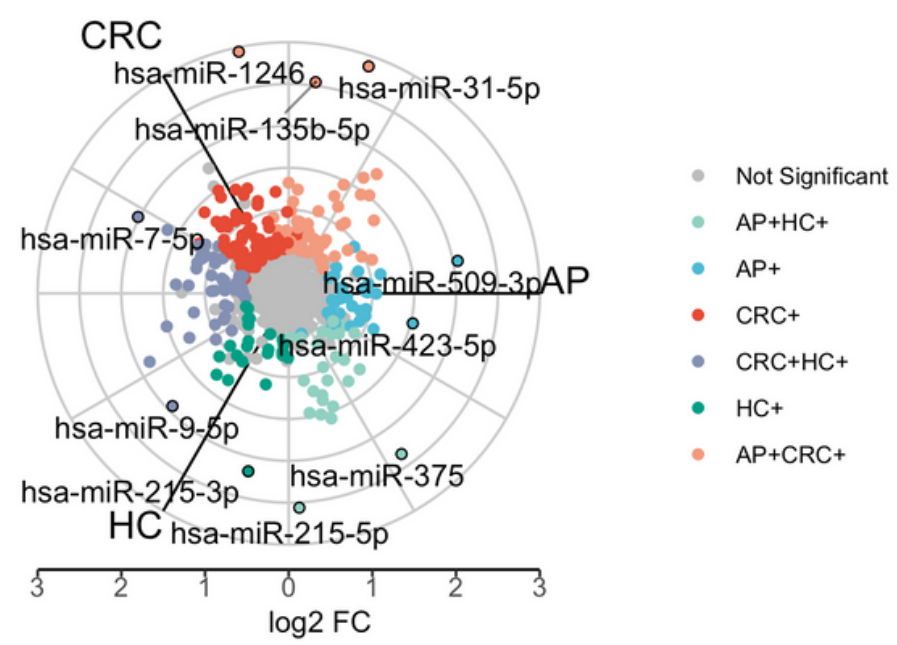

C

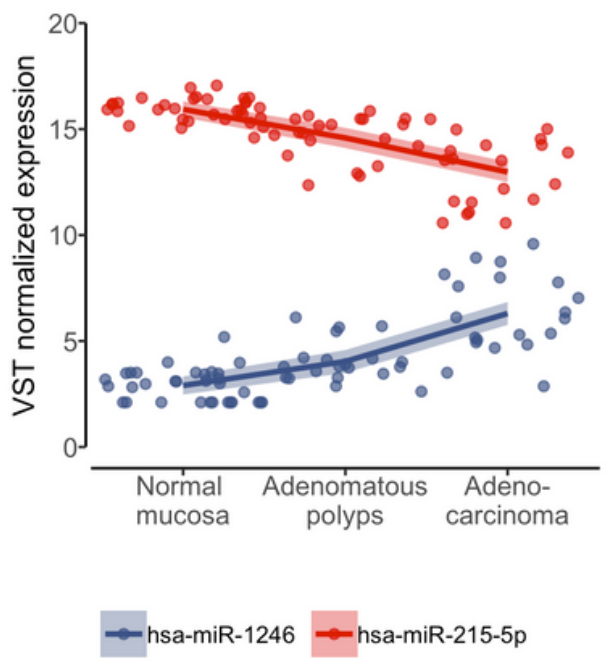

D

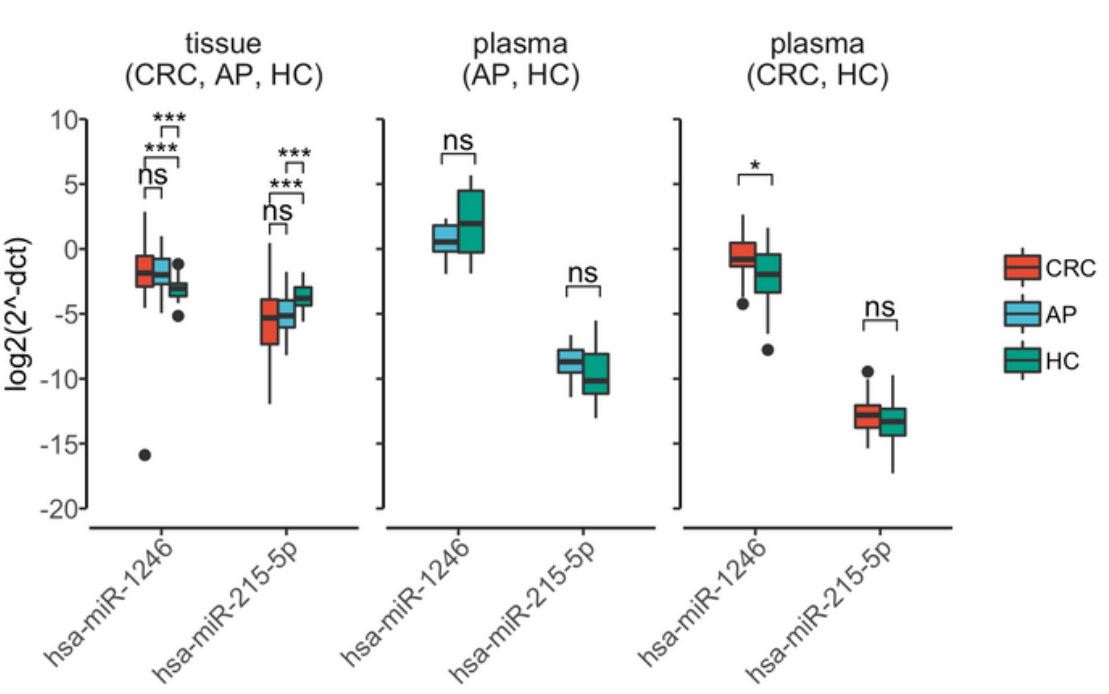

\section{Figure 1}

Expression profiling of miRNAs in precancerous and cancerous tissues of the colon. A: MDS plot showing three clearly resolved clusters corresponding to colorectal cancer (CRC), adenomatous polyps (AP) patients and healthy controls (HC). The analysis was performed on normalized miRNA count data using Euclidean distance. B: A radar plot showing commonly and uniquely 
deregulated miRNAs (PFDR $<0.01$ and $|\log 2 \mathrm{FC}|>0.5$ ) among CRC, AP and HC groups (indicated by colors) in small RNA-seq data. C: miRNAs highly correlating (absolute value of rSpearman's $>0.7$ ) with the stages of healthy to adenoma-carcinoma sequence. Spearman's rank correlation analysis was performed on variance stabilizing transformed miRNA counts. D: The boxplots display expression levels (delta Ct) of hsa-miR-1246 and hsa-miR-215-5p measured by RT-qPCR in tissue and plasma samples of an independent cohort of HC, AP and CRC individuals. Due to the use of different RT-qPCR assays, the results of AP and CRC plasma samples are displayed separately. The $\triangle \mathrm{Ct}$ values were inversed in order to show true direction of the expression. Significance levels: *PFDR $<0.05$; ** PFDR $<0.001$; ns - not significant.

A

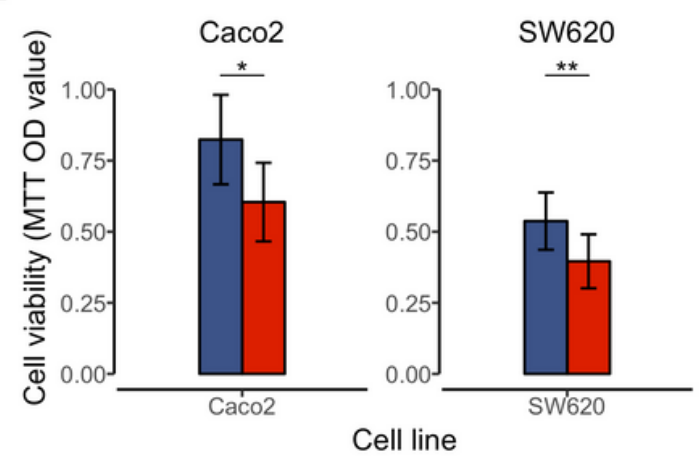

C

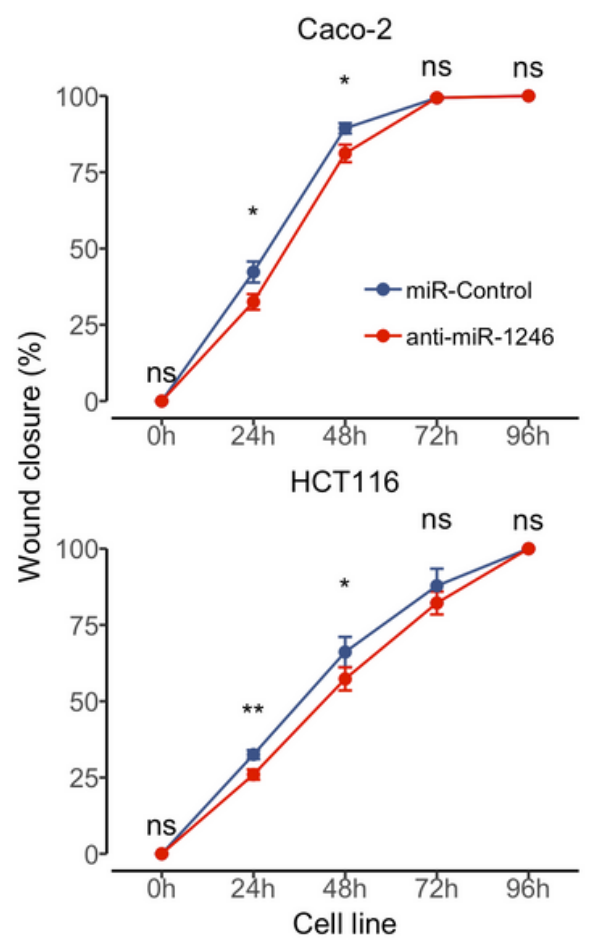

\section{B}

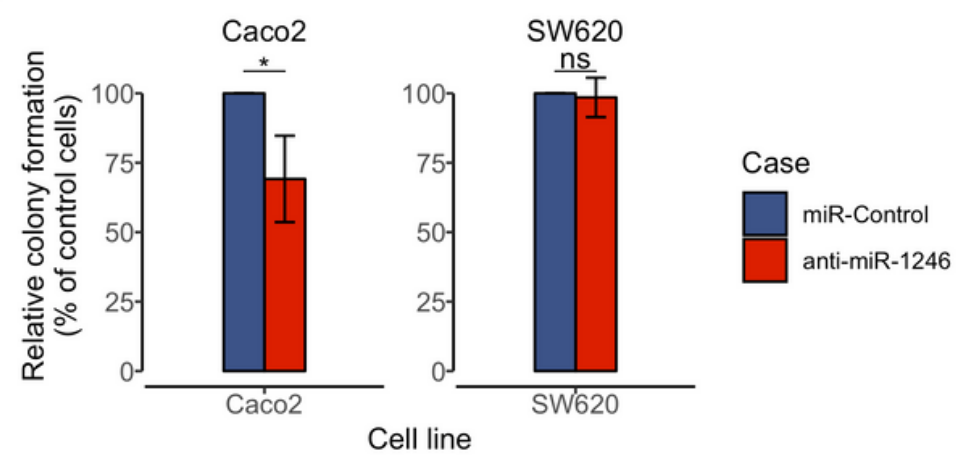

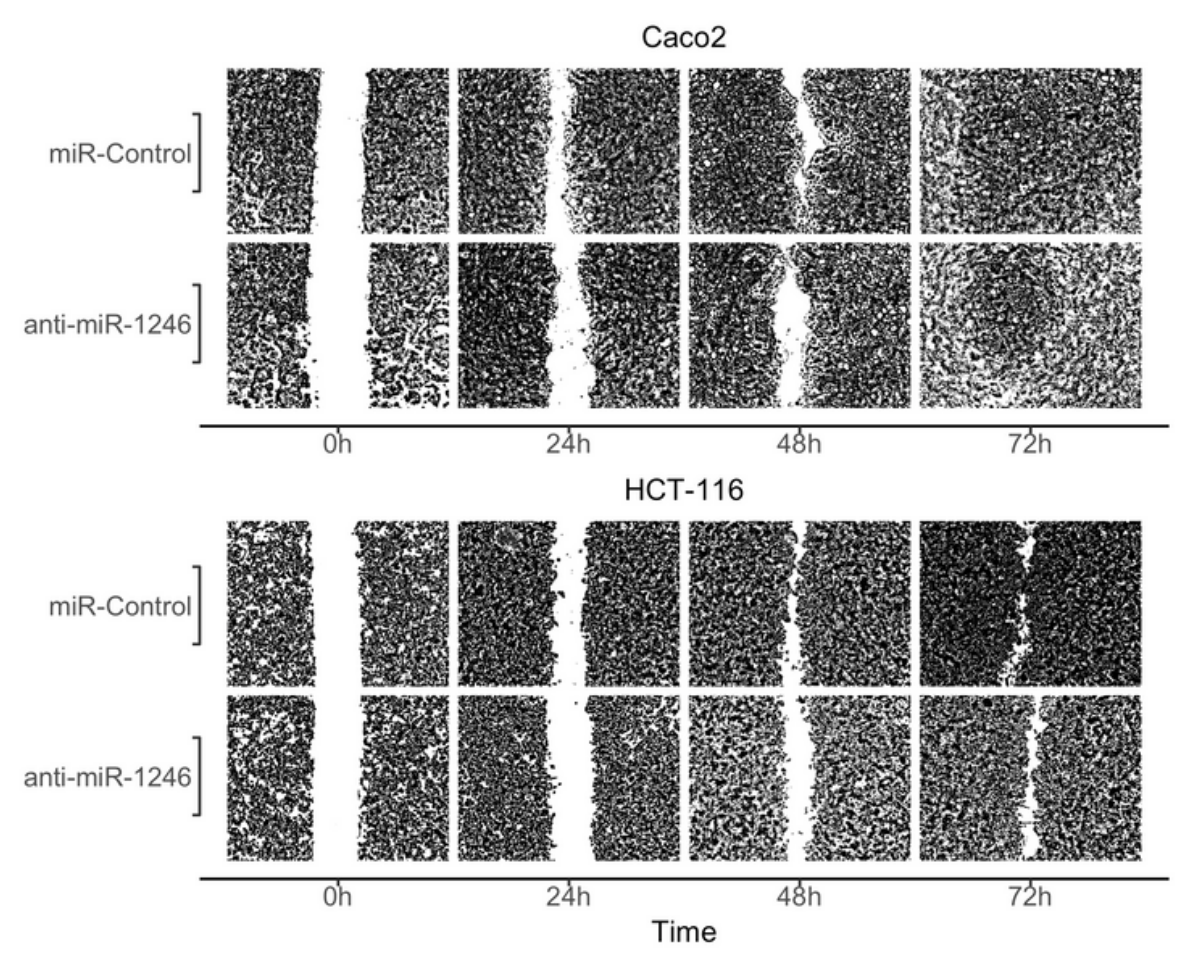

Figure 2

Effect of hsa-miR-1246 inhibition on cell viability, colony formation and migration of Caco-2, HCT116 and SW620 cells. Inhibitor anti-miR-1246: A: reduced cell viability in Caco-2 and SW620 cells; B: reduced colony formation ability in Caco-2 cells; C: lowered cell migration rates in Caco-2 and HCT116 cells $24 \mathrm{~h}$ and $48 \mathrm{~h}$ after transfection. Image magnification is $\times 20$. Data are presented as the mean \pm standard deviation (SD) of three independent experiments. Significance levels: *PFDR $<0.05$; $\star \star P F D R<$ 0.01 ; ns - not significant. Since wounds were fully closed after $96 \mathrm{~h}$, images representing this time point were not included in the figure. 

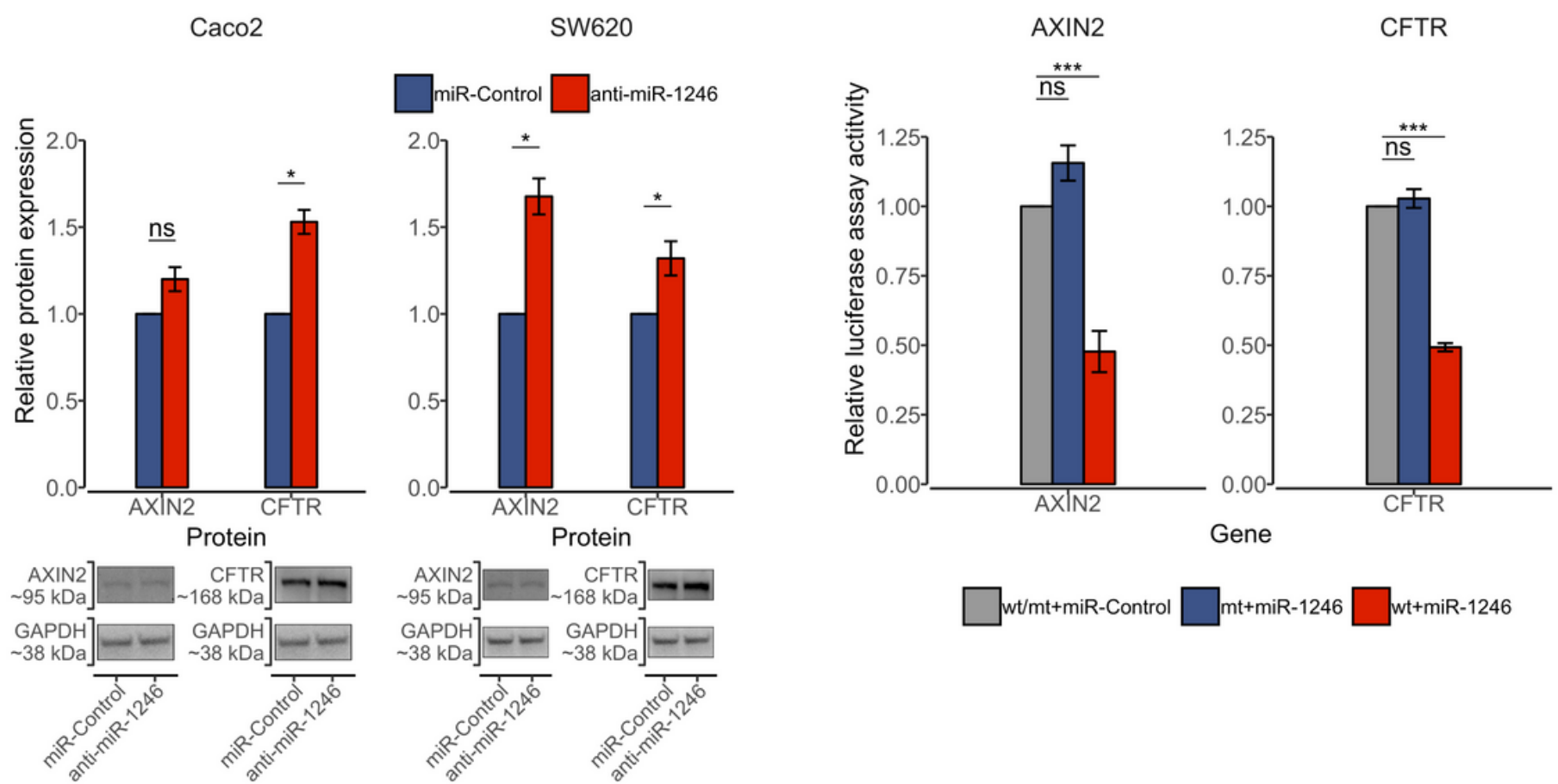

B

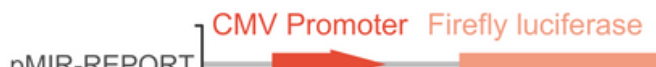

Target 3'UTR SV40 Poly(A)

$$
\begin{array}{r}
\text { CFTR wt } \\
\text { (3'UTR pos 1537-1544) } \\
\text { CFTR mtt } \\
\text { (3'UTR pos 1537-1544) } \\
\text { AXIN2 wt } \\
\text { (3'UTR pos 313-320) } \\
\text { AXIN2 mt } \\
\text { (3'UTR pos 313-320) }
\end{array}
$$

hsa-miR-1246]

\author{
5'-UAAAAUAUCACUUGUCAAUA AAAUCCAU ACAUUUGUGUgaAAUGCAGAA-3' \\ 5'-UAAAAUAUCACUUGUCAAUA AAAUUUAU ACAUUUGUGUGAAAAUGCAGAA-3' \\ 5'-UAUGACUUCCUAGGGAUCUG AAAUCCAU AAACUAAGAGAAACUGUGUAUA-3' \\ 5'-UAUGACUUCCUAGGGAUCUG AAAUUUAU AAACUAAGAGAAACUGUGUAUA-3'
}

\section{Figure 3}

hsa-miR-1246 suppresses expression of CFTR and AXIN2 via direct 3'UTR targeting. A: Effect of hsa-miR-1246 inhibition on CFTR and AXIN2 protein expression compared to negative control (miR-Control) measured at $72 \mathrm{~h}$ after transfection of Caco-2 and SW620 cells. Protein bands representing the signals detected by Western blot are provided at the bottom of the panel. Protein expression was normalized to the expression values of GAPDH reference protein. Data from three to four independent experiments presented as the mean of relative protein expression \pm standard deviation (SD). B: Representation of constructed pMIR-REPORT vectors containing wild type (wt) and mutant type (mt) 3' UTR sequences of AXIN2 and CFTR genes, that are within predicted binding sites of hsa-miR-1246. C: Downregulation of AXIN2 and CFTR by hsa-miR-1246 mimics. Luciferase activity of cells transfected with negative controls (miR-Control) was used as a normalization factor and was set at $100 \%$. Data are presented as the mean \pm SD of three independent experiments. Luciferase activity significantly decreased in co-transfection with hsa-miR-1246 mimic and wild-type 3'-UTR-luciferase plasmid of both CFTR and AXIN2 target genes after $72 \mathrm{~h}$ Significance levels: *PFDR $<0.05$; ***PFDR $<0.001$; ns - not significant.

\section{Supplementary Files}

This is a list of supplementary files associated with this preprint. Click to download. 
- SupplementaryFigures.docx

- SupplementaryTable1.xIsx

- SupplementaryTable2.xIsx

- SupplementaryTable3.xIsx

- SupplementaryTable4.xlsx

- WesternBlotsupplementarymaterial.pdf 